Research Article

\title{
COMPARISON OF ECO-MSE AND PST-MSE PRIMER COMBINATIONS IN GENERATING AFLP MAP OF TOMATO
}

\author{
B. R. Ojha \\ Institute of Agriculture and Animal Sciences, Rampur, Chitwan, Nepal
}

\begin{abstract}
An experiment was conducted at Wageningen University and Research Centre (WUR), Unifarm, Haarweg, Wageningen, The Netherlands in 2000 to compare Eco-Mse and Pst-Mse primer combinations to generate AFLP markers of tomato. Back cross first $\left(\mathrm{BC}_{1}\right)$ population of Lycopersicon esculentum $\mathrm{cv}$ money maker and Lycopersicon pennellii LA716 was used. Six primer combinations (three of Eco-Mse and three of Pst-Mse) were used. A total of 122 AFLP markers were found generating 76 markers by Eco-Mse primer combination and 46 markers by Pst-Mse primer combination. The average number of informative markers per primer combination was 20.33 ranging from 11 (P11M50) to 36 (E35M48). Similarly the average number of informative markers per chromosome was 10.16 ranging from 4 (chromosome number 8 ) to 16 (chromosome number 1). Within Eco-Mse primer combinations, E32M50 generated the least (18) and E35M48 generated the highest (36) AFLP markers. Similarly, within Pst-Mse primer combination, P14M50 generated the highest (20) and P11M50 generated the least (11) AFLP markers. The Eco-Mse primer combination generated the highest number of marker (12) in chromosome 9 and the lowest (2) in chromosome number 8. Similarly, the Pst-Mse primer combination generated the highest number of markers ( 9 ) in chromosome number 1 and the lowest (2) in chromosome number 3, 8, 11 and 12. The AFLP map spanned $843 \mathrm{cM}$. The longest AFLP map was found in chromosome 1 and spanned $98 \mathrm{cM}$ and the shortest in chromosome 8 and spanned $46 \mathrm{cM}$.
\end{abstract}

Key words: Tomato, Back cross, Primer combination, AFLP map

\section{INTRODUCTION}

In Nepal, vegetable crops are grown in 181,000 ha of land and the production is achieved by $2038,000 \mathrm{mt}$ that contributes $5.73 \%$ to the agricultural gross domestic products (MOAC, 2005). Tomato (Lycopersicon esculentum) is the most important and widely grown vegetable crop in the world. It is a day neutral crop and is found in both determinate and indeterminate types. The indeterminate types are grown in greenhouses whereas the determinate types are grown in field as well as greenhouse. Tomato is commercially important throughout the world in food processing industries. The indeterminate type is grown for fresh fruit market.

The progress in plant breeding is easy and quick for qualitative characters controlled by major genes whereas quantitative characters like yield, earliness, days to heading, stress tolerance, rate of development, quality, adaptation and height etc is still more complicated. These traits are controlled by several genes that have cumulative effects (Van Berloo, 2000). Quantitative Trait Loci (QTLs) harbours such genes and plant breeders are interested to find such genes that could efficiently help in crop improvement. DNA fingerprinting and QTL mapping are the powerful tools to unravel the genetic basis of polygenic traits. Creation of genetic variation and then selection of suitable genotypes is one of the common ways that can assist in crop improvement. It is becoming easier to enhance the exploitation of the germplasm of crop species with the advent of molecular markers like Amplified Fragment Length Polymorphism (AFLP), Restriction Fragment Length Polymorphism (RFLP), Random Amplified Polymorphic DNA (RAPD), Sequence Tagged Site (STS) and so on. These molecular or DNA markers have many applications in plant breeding such as estimation of genetic diversity, characterisation of germplasm resources, identification of crop varieties and marker assisted selection. The marker-assisted selection has been utilised in gene introgression, elimination of linkage drag and gene pyramiding. Successful pyramiding of genes $\mathrm{Xa} 4$, Xa5, Xa13 and Xa21 conferring resistance to 4 races of bacterial leaf blight pathogen has been achieved in rice (Huang et at, 1997) by using molecular markers, which was otherwise impossible through conventional methods.

Evidences have been obtained that wild species of Lycopersicon have alleles associated with desirable traits 
(Bernacchi et al., 1998; Vicente and Tanksley, 1993; McNally and Mutschler, 1997; Fulton et al., 1997; Fulton et al., 2000). Various researches were carried out to find valuable QTLs for different characters in crop plants. In wild relatives of tomato, QTLs were found for valuable traits (Paterson et al., 1988; Lefort et al., 1990; Alpert et al., 1995; Doganlar et al., 2000; Thoquet et al., 1996; Carlos, 1998; Foolad et al., 1998; Fulton et al., 1997; McNally and Mutschler, 1997).

AFLP technique is one of the most efficient and realistic techniques used in generating linkage map because it combines the advantages of RFLPs and RAPDs with high reproducibility and locus specific. Lycopersicon esculentum cv Money Maker is a high yielding cultivar whereas Lycopersicon pennellii is a wild species that has genes for increase in yield (Vicente and Tanksley, 1993) and insect resistance (McNally and Mutschler, 1997).

The Eco-Mse and Pst-Mse primer combinations are used in generating AFLP map. These primer combinations produce more AFLP markers than other combinations. Pst-Mse AFLP markers are found in the distal part of the chromosomes. The majority of the Eco-Mse AFLP markers mapped in clusters are found in the central part of the chromosomes. If the markers are distributed evenly in the entire chromosomes, a large genome can be found. In past most of the researches were mainly carried out with regard to varietal adaptation, characterisation of germplasm and study of different traits (Upreti and Adhikary, 1984; Upreti et al., 1985; Shakya et al., 1993; Ojha, 2004). But some researches were directed towards this approach and satisfactory results were found (Ojha, 2001; Ojha, 2003; Haanstra, 2000; and Vos et al., 1995). To give continuity in this area and to verify the previous findings, this study was conducted to compare Eco-Mse and Pst-Mse primer combinations in generating AFLP map of tomato that helps in finding QTLs important for crop improvement.

\section{MATERIALS AND METHODS}

AFLP map of tomato was developed from $44 \mathrm{BC}_{1}$ populations. To obtain this map, DNA was extracted from leaves and AFLP data was analysed. Then with the help of JoinMap 2.0 program an AFLP map was constructed. The detail procedure in generating AFLP map is explained below.

\section{Plant material}

Forty four plants in the Backcross first $\left(\mathrm{BC}_{1}\right)$ population of the interspecific cross Lycopersicon esculentum $\mathrm{cv}$ Money Maker X Lycopersicon pennellii LA716 were genetically analysed with the help of AFLP markers.

\section{Experimental design}

The research was carried out in the Unifarm, Haarweg, Wageningen, The Netherlands. Plants were grown under the conditions that are similar environments to those in commercial greenhouse tomato cultivation. The $\mathrm{BC}_{1}$ population seeds were sown in trays filled with a commercial potting soil mixture. After three weeks, the seedlings were transferred to rockwool and placed on benches. The plants were grown by giving $100 \mathrm{~cm}$ row to row distance and $50 \mathrm{~cm}$ plant to plant distance. After every two $\mathrm{BC}_{1}$ plants, one recurrent parent Money Maker was planted to facilitate the crossing.

\section{Isolation of DNA from leaves}

The protocol for the isolation of DNA was used according to the standard tomato DNA isolation protocol developed in the Laboratory of Plant Breeding, Wageningen University and Research Centre (WUR). Young leaf of $2-3 \mathrm{~cm}^{2}$ of each $\mathrm{BC}_{1}$ plant was collected. The sample of leaf was crushed with the help of grinder. Four hundred $\mu \mathrm{l}$ extraction buffer, $500 \mu \mathrm{l}$ nuclei lysis buffer and $50 \mu \mathrm{l}$ sarkosyl were added. Then the samples were shaked and vortexed and incubated in a water bath at $65^{\circ} \mathrm{C}$ for one hour. The tubes were taken out and $800 \mu \mathrm{l}$ chloroform and isoamylalcohol in 24:1 ratio was added to each sample. Centrifuging was done and supernatant was pipetted. Eight hundred $\mu \mathrm{l}$ isopropanol was added to each sample. Pellet was seen after centrifuging and rinsed for $20 \mathrm{~min}$ in $250 \mu \mathrm{l} 76 \%$ ethylalcohol with $10 \mathrm{mM}$ ammonium acetate (NH4Ac). The pellets were dried and dissolved in $100 \mu \mathrm{l}$ tris EDTA (TE) buffer (+ $1 \mu \mathrm{g}$ Rnase). 


\section{The amplified fragment length polymorphism (AFLP) protocol}

The AFLP procedure as described by Vos et al (1995) was applied to perform the experiment. The adapters and primers used for this experiment are listed in Table 1. The primer combinations: E32M49, E32M50, E35M48, P11M50, P14M49 and P14M50 were used. Most informative primer combinations were selected from a previous study done by Haanstra, 2000 and Vos et al.; 1995.

Table 1. List of primers and adapters

\begin{tabular}{|c|c|}
\hline Primers/Adapters & Sequences \\
\hline Mse 1 Adapter & $\begin{array}{c}\text { 5'-GACGATGAGTCCTGAG-3' } \\
\text { 3'-TACTCAGGACTCAT-5' }\end{array}$ \\
\hline MOO (Universal primer) & GATGAGTCCTGAGTAA \\
\hline \multicolumn{2}{|l|}{ Mse1 +3 primers } \\
\hline M48 & $\mathrm{MOO}+\mathrm{CAC}$ \\
\hline M49 & $\mathrm{MOO}+\mathrm{CAG}$ \\
\hline M50 & $\mathrm{MOO}+\mathrm{CAT}$ \\
\hline EcoR1 Adapter & $\begin{array}{r}\text { 5'-CTCGTAGACTGCGTACC-3' } \\
\text { 3'-CTGACGCATGGTTAA-5' }\end{array}$ \\
\hline EOO (Universal primer) & GACTGCGTACCAATTC \\
\hline \multicolumn{2}{|l|}{ EcoR1 +3 primers } \\
\hline E32 & $\mathrm{EOO}+\mathrm{AAC}$ \\
\hline E35 & $\mathrm{EOO}+\mathrm{ACA}$ \\
\hline Pst1 Adapter & $\begin{array}{l}\text { 5'-CTCGTAGACTGCGTACATGCA-3' } \\
\text { 3'-CATCTGACGCATGT-5' }\end{array}$ \\
\hline POO (Universal primer) & GACTGCGTACATGCAG \\
\hline \multicolumn{2}{|l|}{ Pst +2 primers } \\
\hline P11 & $\mathrm{POO}+\mathrm{AA}$ \\
\hline P14 & $\mathrm{POO}+\mathrm{AT}$ \\
\hline
\end{tabular}

AFLP data analysis and Chi-square of homogeneity test

Each sample containing $0.6 \mu \mathrm{l}$ was loaded in the licor gel with the help of micro pipette. The samples of Lycopersicon esculentum $\mathrm{cv}$ Money maker and Lycopersicon pennellii were kept as control. After running licor gel, the AFLP picture of forty four $\mathrm{BC}_{1}$ plants was taken out. AFLP markers were scored dominantly and visually with the help of developed picture. The scoring was tabulated giving symbols $\mathrm{H}$ for heterozygousness of L. pennellii specific, A for homozygousness of Money Maker specific and U for doubtful bands. Chi square of homogeneity test between two groups as well as within group of primer combinations was performed.

\section{Map construction}

The computer programme JoinMap 2.0 (Stam and Van Ooijen 1996) was used to construct an AFLP map. The modules JMGRP 32, JMTREE 32, JMSPL 32, JMSLA 32, JMREC 32 and JMMAP 32 were used. For calculating mapping distances, Kosambi's maping function was used. Likewise 0.1, 0.49, 5, 3 and 7 values were used as LOD threshold, recombination threshold, jump value, ripple value and triplet threshold respectively. The Map Chart software programme developed by Voorrips 1999, was used for drawing the map of the chromosomes. The data was scored visually with the help of developed pictures of $44 \mathrm{BC}_{1}$ plants (Table 2). These data were used in constructing AFLP map.

\section{RESULTS AND DISCUSSION}

\section{Analysis of AFLP markers}

Based on Haanstra, 2000 findings, 6 informative primer combinations (3 Eco-Mse and 3 Pst-Mse) were used for this research. AFLP markers were evaluated in back cross 1 population of Lycopersicon esculentum X Lycopersicon pennellii. The AFLP map was constructed based on pennellii specific markers.

A total of 122 markers were found in this experiment. Seventy six markers was found in Eco-Mse primer combination and 46 markers in Pst-Mse primer combination. The number of AFLP markers generated by different Eco-Mse and Pst-Mse primer combinations in different chromosomes is presented in Table 2. 
Table 2. The number of AFLP markers generated by different Eco-Mse and Pst-Mse primer combinations in different chromosomes

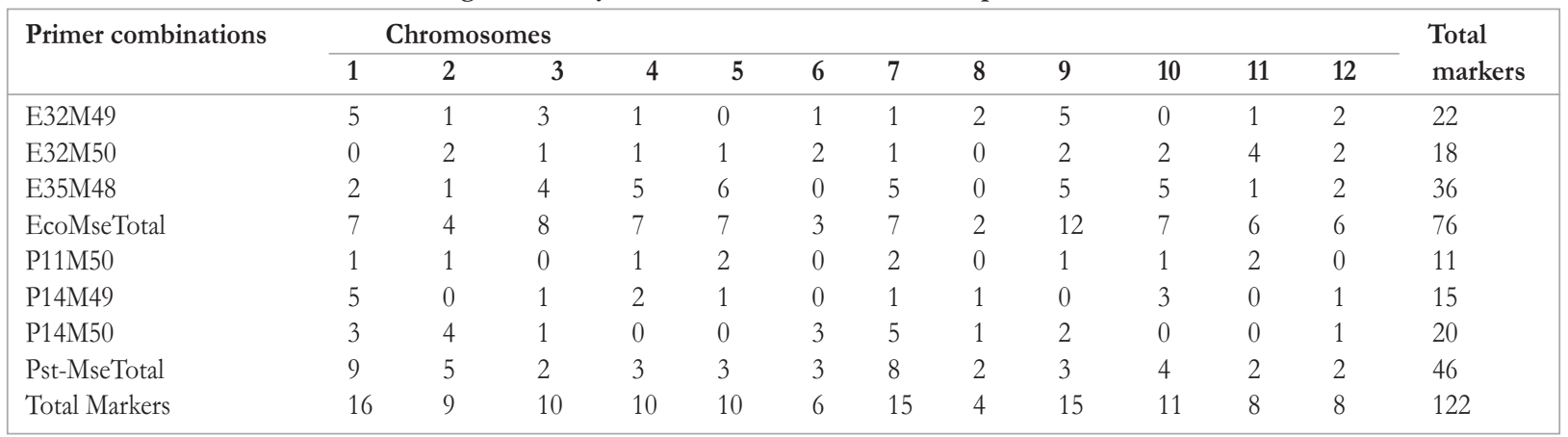

The primer combination E35M48 generated the highest number of markers (36). On the other hand, P11M50 generated the least number of markers (11). The AFLP markers were found the highest number (16) in chromosome 1 whereas the least (4) in chromosome 8. In chromosome 9, the Eco-Mse primer combination produced the highest number of marker (12). In constrast, this primer combination produced the least marker (2) in chromosome 8. Similarly, the Pst-Mse primer combination generated the highest number of markers (9) in chromosome 1 and the least (2) in chromosomes 3, 8, 11 and12.

The Eco-Mse primer combination produced in equal markers (7) in chromosomes 1, 4, 5, 7 and 10, and 6 markers in each chromosome 11 and 12. Likewise, the Pst-Mse primer combination produced equal markers (3) in chromosomes 4, 5, 6 and 9, and 2 markers in each chromosome 3, 8, 11 and 12. The Eco-Mse and PstMse combination generated equal number of markers ( 3 and 2) in chromosome 6 and 8 respectively.

Equal number of markers (10) were found in chromosome 3, 4 and 5; 8 markers in chromosome 11 and 12; and 15 markers in chromosome 7 and 9. E32M49 produced no markers in chromosome 5 and 10 and produced equal markers in chromosomes 2, 4, 6, 7 and 11. No markers in chromosome 1 and 8 were produced by E32M50 and generated equal marker (1) in chromosomes 3, 4, 5 and 7 and 2 markers in chromosomes 2, 6, 9, 10 and 12. Similarly, E35M48 produced no markers in chromosomes 6 and 8 but generated equal markers (5) in chromosomes 4, 7, 9 and 10; 2 markers in chromosomes 1 and 12; 1 marker in chromosome 2 and 11. Likewise, the primer combination P11M50 produced no markers in chromosomes 3, 6, 8, 12 but generated equal markers (1) in chromosomes 1,2, 4, 9 and 10 and 2 markers in chromosomes 5, 7 and 11. Similarly, the primer combination P14M49 produced no markers in chromosomes 2, 6, 9, and 11 but generated equal markers (1) in chromosomes 3, 5, 7, 8 and 12. Likewise, the primer combination P14M50 also produced no markers in chromosomes 4, 5, 10 and 11 but generated equal markers (3) in chromosomes 1 and 6; 1 marker in chromosomes 3, 8 and 12. All primer combinations produced markers in chromosome 7. P14M49 and P14M50 generated no markers in chromosome 11 but generated equal markers in chromosomes 3, 8 and 12. All Eco-Mse primer combinations produced same number of marker (2) in chromosome 12. Two primer combinations E35M48 and P11M50 produced no markers in chromosomes 6 and 8. Similarly, two primer combinations namely E32M49 and P14M50 produced no markers in chromosomes 5 and 10. The average number of markers per Eco-Mse primer combination was 25.3, and per Eco-Mse primer combination per chromosome was 6.3. Similarly, the average number of markers per Pst-Mse primer combination was 15.3 whereas per Pst-Mse primer combination per chromosome was 3.8. In chromosome number 2, 6, 8, 11 and 12, the Eco-Mse primer combination generated less AFLP markers than average (6.3). Similarly, in chromosomes 3, 4, 5, 6, 8, 9, 11 and 12 the Pst-Mse primer combination generated less AFLP markers than average (3.8). Both primer combinations Eco-Mse and PstMse generated more markers in chromosomes 1, 7 and 10 than their respective averages.

As a whole, the Eco-Mse primer combination produced more AFLP markers than the Pst-Mse primer combination. This findings was in agreement with the findings of Haanstra, 2000; Carlos, 1998; Vos et al., 1995 and Ojha, 2001.

\section{Chi-square test}

The AFLP markers produced by Eco-Mse and Pst-Mse primer combinations was observed in 76 and 46 numbers respectively. It was assumed that the two groups of primer combinations would produce the same 
proportion of markers. On the basis of chi-square test, Eco-Mse primer combination appeared significantly different from Pst-Mse primer combination at $5 \%$ and $1 \%$ level of significance for $1 \mathrm{df}$. It was shown that both groups of primer combinations did not generate the same proportion of AFLP markers (Table 3). Within group of Eco-Mse primer combination, equal proportion of AFLP markers were not generated by Eco-Mse primers whereas within group of Pst-Mse primer combination, equal proportion of AFLP markers were generated by Pst-Mse primers on the basis of chi square test (Table 3). This findings is seemed similar to the findings of many authors (Haanstra, 2000; Carlos, 1998; Paterson et al., 1998).

Table 3. Chi-square of homogeneity test within and between groups of primer combinations

\begin{tabular}{|c|c|c|c|c|c|c|}
\hline \multirow{2}{*}{$\begin{array}{l}\text { SN } \\
1\end{array}$} & \multirow{2}{*}{$\begin{array}{l}\text { Particulars } \\
\text { Both groups }\end{array}$} & \multicolumn{2}{|c|}{ AFLP Markers } & \multirow{2}{*}{$\begin{array}{l}\chi^{2} \text { 'Cal' } \\
\text { value }\end{array}$} & \multirow{2}{*}{$\frac{\chi^{2}}{\text { ‘tab’ at } 5 \%}$} & \multirow{2}{*}{$\begin{array}{r}\chi^{2} \\
\text { 'tab' at 1\% }\end{array}$} \\
\hline & & Expected & Observed & & & \\
\hline & Eco-Mse & 61 & 76 & 3.69 & & \\
\hline & Pst-Mse & 61 & 46 & 3.69 & & \\
\hline & Sum of $\chi^{2}$ & & & $7.3 * *$ & 3.84 & 6.63 \\
\hline \multirow[t]{5}{*}{2} & Eco-Mse group & & & & & \\
\hline & E32M49 & 25.33 & 22 & 0.44 & & \\
\hline & E32M50 & 25.33 & 18 & 2.12 & & \\
\hline & E35M48 & 25.33 & 36 & 4.49 & & \\
\hline & Sum of $\chi^{2}$ & & & 7.05* & 5.99 & 9.2 \\
\hline \multirow[t]{5}{*}{3} & Pst-Mse group & & & & & \\
\hline & P11M50 & 15.33 & 11 & 1.22 & & \\
\hline & P14M49 & 15.33 & 15 & 0.01 & & \\
\hline & P14M50 & 15.33 & 20 & 1.42 & & \\
\hline & Sum of $\chi^{2}$ & & & $2.65^{\mathrm{ns}}$ & 5.99 & 9.2 \\
\hline
\end{tabular}

\section{Map construction}

The computer programme JoinMap 2.0 (Stam and Van Ooijen 1996) was used to construct an AFLP map. The Map Chart software programme developed by Voorrips 1999, was used for drawing the map of the chromosomes. The longest AFLP map was found in chromosome 1 and spanned $98 \mathrm{cM}$ whereas the shortest was found in chromosome 8 and spanned $46 \mathrm{cM}$.

It was observed that vast majority of Eco-Mse markers occurred in clusters. In chromosomes 1, 3, 4, 5 and 7, majority of the Eco-Mse AFLP markers were clustered in distances between 25-28 cM, 15-21 cM, 26-39 cM, 24-36 cM and 14-30 cM respectively. Similarly, in chromosomes 9, 10, 11 and 12 these markers were clustered in distances between 18-19 cM, 20-24 cM, 47-49 cM and 14-16 cM respectively. On the other hand, Pst-Mse markers were not occurred in clusters. Eco-Mse markers were found in the central part of the chromosomes but the Pst-Mse markers were found in the distal part of the chromosomes. This result supported the previous findings of many author (Ojha, 2001; Haanstra, 2000; Paterson, 1998; Carlos, 1998). The map of the chromosomes is given in Figure 1. 


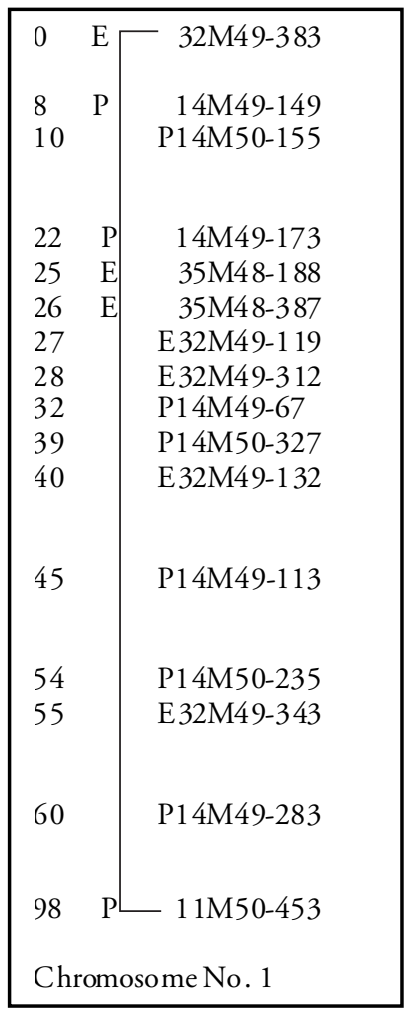
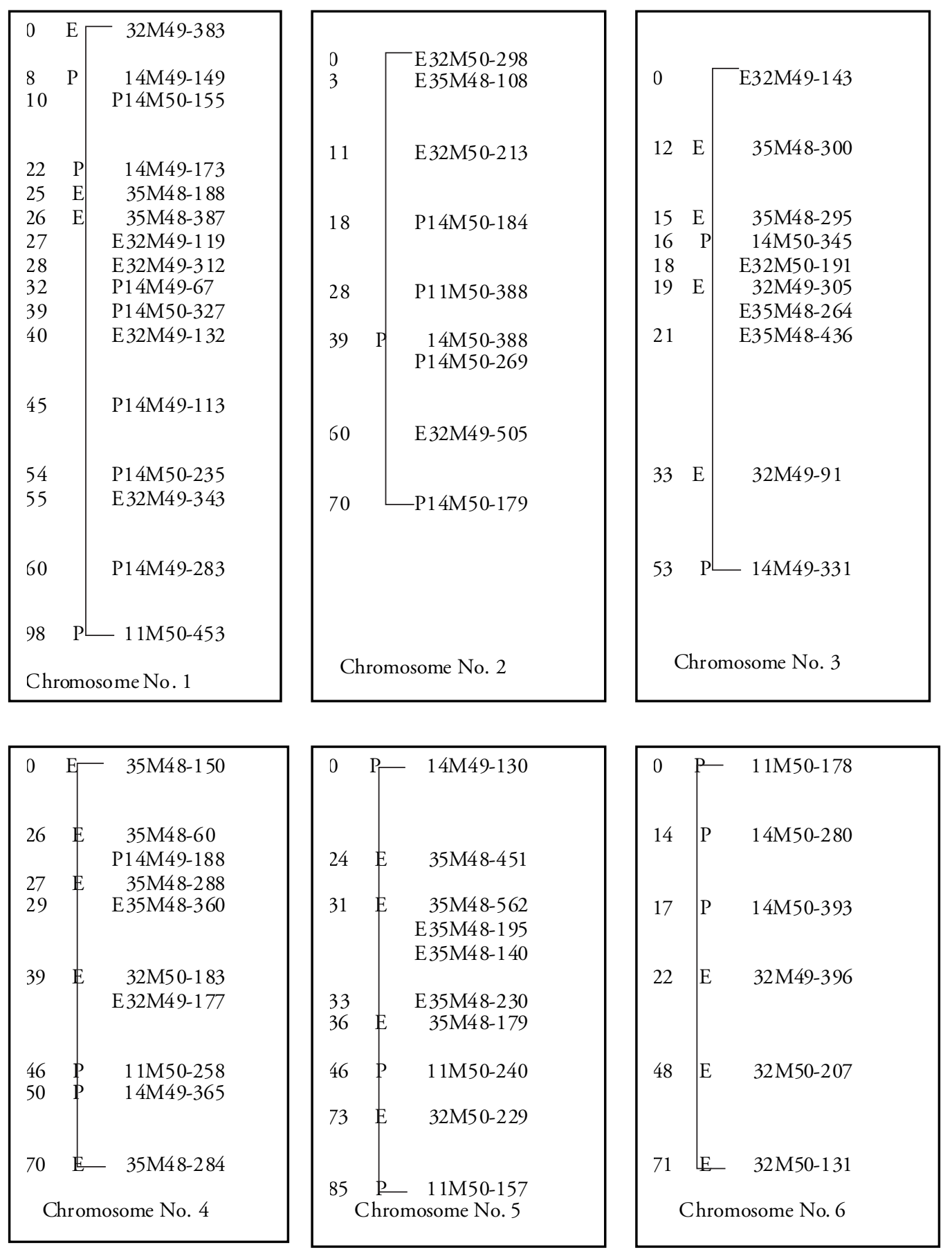

Figure 1. Map of chromosomes 
Figure 1. Contd......

\begin{tabular}{|ccc|}
\hline & & \\
0 & & P14M50-169 \\
11 & $\mathrm{P}$ & $14 \mathrm{M} 50-313$ \\
& & \\
14 & & E35M48-568 \\
18 & & E32M49-247 \\
19 & & E35M48-88 \\
20 & $\mathrm{E}$ & $32 \mathrm{M} 50-294$ \\
& & E35M48-170 \\
& & \\
23 & & P14M50-167 \\
27 & & E35M48-129 \\
30 & & E35M48-120 \\
& & \\
& & \\
38 & & P11M50-90 \\
41 & P11M50-150 \\
46 & P14M49-268 \\
52 & P14M50-256 \\
& & \\
70 & P14M50-220 \\
Chromosome No. 7
\end{tabular}
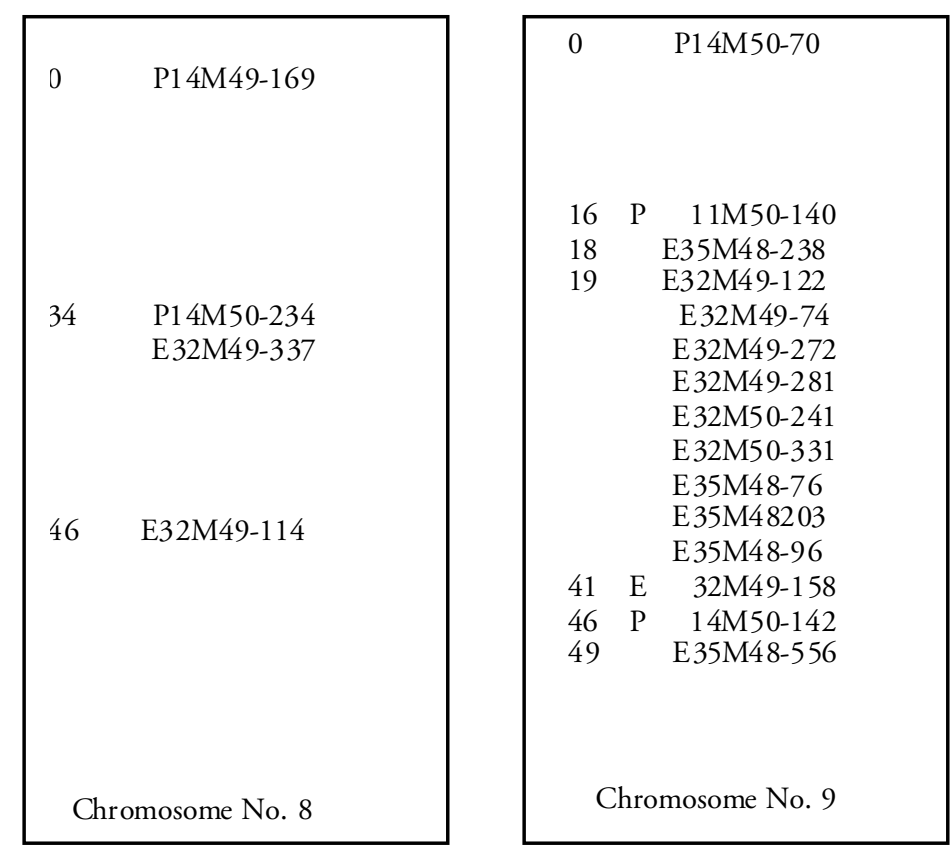

\begin{tabular}{|cc|}
\hline 0 & P14M49-157 \\
& \\
19 & P14M49-148 \\
20 & E32M50-343 \\
22 & E35M48-136 \\
& E35M48-212 \\
& E35M48-315 \\
24 & E32M50-250 \\
& E35M48-439 \\
& \\
31 & P11M50-510 \\
& \\
41 & E35M48-371 \\
& \\
& \\
75 & \\
& \\
Chromosome No. 10
\end{tabular}

\begin{tabular}{|ll|}
\hline 0 & P11M50-228 \\
15 & P11M50-276 \\
47 & E32M50-493 \\
48 & E32M50-245 \\
49 & E35M48-216 \\
58 & E32M50-172 \\
66 & E32M50-121 \\
& \\
& \\
& \\
& \\
& \\
& \\
&
\end{tabular}

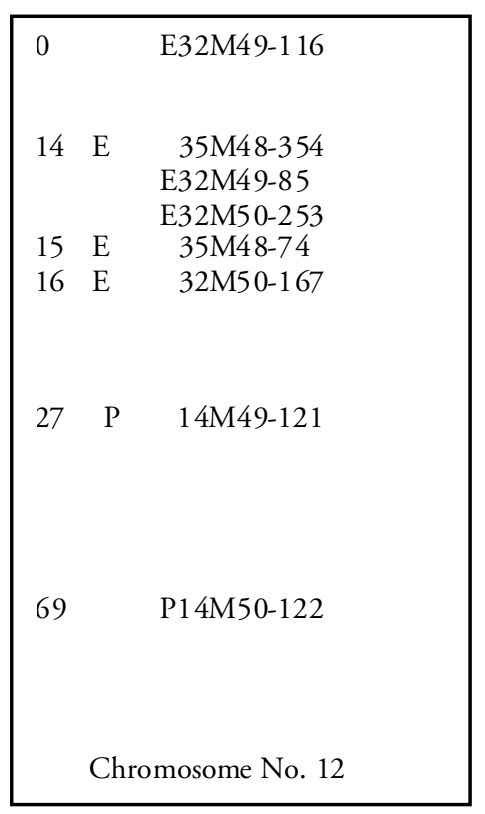

\section{CONCLUSIONS}

The progress in plant breeding is easy and quick for qualitative characters controlled by major genes with easily identifiable effect on the phenotype. The identification of desirable genotypes for the quantitative characters is still more complicated. DNA fingerprinting and QTL mapping are the powerful tools to unravel the genetic basis of quantitative traits. Making distant crosses of complementary genotypes creates the new genetic variation. Molecular markers AFLP, RFLP, RAPDs and STS have many applications in plant breeding. The markerassisted selection has been utilised in gene introgression, elimination of linkage drag and gene pyramiding. Most of the wild species of tomato have desirable characters, like insect resistance, disease resistance, firmness and earliness that can be introduced to the elite cultivated species. So these wild species are widely used in crossing programme because of the certain agriculturally important genes. These genes are easily and quickly trapped with the help of molecular markers. 
A total of 122 AFLP markers were found generating 76 markers by Eco-Mse primer combination and 46 markers by Pst-Mse primer combination. The Eco-Mse primer combinations produced more markers than PstMse combination. The average number of informative markers per primer combination was 20.33 ranging from 11 (P11M50) to 36 (E35M48). The average number of informative markers per chromosome was 10.16. The Eco-Mse primer combination generated the highest number of marker (12) in chromosome nine and PstMse primer combination generated the highest number of markers (9) in chromosome number one. The majority of Eco-Mse markers occurred in clusters but not the Pst-Mse markers. Eco-Mse markers were found in the central part of the chromosomes but the Pst-Mse markers were found in the distal part of the chromosomes. More number of AFLP markers are obtained using Eco-Mse and Pst-Mse primer combinations and then a large genome can be found. When there is large genome, the chance of obtaining many QTLs is high. QTLs harvor such genes that govern agriculturally important traits and plant breeders are interested to grap these genes. A precise genetic map is obtained if the distribution of markers is evenly over the entire genome.

\section{ACKNOWLEDGEMENTS}

This research was supported by Wageningen University and Research Centre, The Netherlands. So, I am highly grateful to this University. Similarly, I am indebted to my supervisors (Prof.Dr.Ir. P. Stam, Dr. Theo Hendriks and Mr. Sylvestre Manga Owana) for their proper guidance and help to complete this research. I would also like to express my heart felt thanks to all faculties, staff members, colleagues and friends of Department of Plant Breeding for their kind help, co-operation and friendly behaviour.

\section{REFERENCES CITED}

Alpert, K. B., S. Grandillo, and S. D. Tanksley. 1995. A major QTL controlling fruit weight is common to both red and green fruited tomato species. J. Theor. Appl. Genet. 91: 994-1000.

Bernacchi, D., T. Beck-Bunn, Y. Eshed, J. Lopez, V. Petiard, J. Uhlig, D. Zamir, and S. D. Tanksley. 1998. Advanced backcross QTL analysis in tomato. I. Identification of QTLs for traits of agronomic importance from Lycopersicon birsutum. J. Theor. Appl. Genet. 97: 381-397.

Carlos L. D. T. E. 1998. Mapping of bacterial wilt resistance genes in tomato (Lycopersicon esculentum) using AFLP (Amplified Fragment Length Polymorphisms). College, Laguna, Philippines.

Doganler, S. S. D. Tanksley, and M. A. Mutschler. 2000. Identification and molecular mapping of loci controlling fruit ripening time in tomato. J. Theor. Appl. Genet.100: 249-255.

Foolad, M. R., F. Q. Chen, and G. Y. Lin. 1998. RFLP mapping of QTLs conferring cold tolerance during seed germination in an interspecific cross of tomato. Molecular Breeding 4: 519-529.

Fulton, T. M., T. Beck-Bunn, D. Emmatty, Y. Eshed, J. Lopez, V. Patiard, J. Uslig, D. Zamir, and S. D. Tanksley. 1997. QTL analysis of an advanced backcross of Lycopersicon perwivinum to the cultivated tomato and comparison with QTLs found in other wild species. J. Theor. Appl. Genet. 95: 881-894.

Fulton, T. M., S. Grandillo, T. Beck-Bunn, E. Fridman, A. Frampton, J. Lopez, V. Patiard, J. Uslig, D. Zamir, and S. D. Tanksley. 2000. Advanced backcross QTL analysis of a Lycopersicon esculentum X Lycopersicon parviflorum cross. J. Theor. Appl. Genet 100: 1025-1042.

Fulton, T. M., J. C. Nelson, and S. D. Tanksley. 1997. Introgression and DNA marker analysis of Lycopersicon peruvianum, a wild relative of the cultivated tomato, into Lycopersicon esculentum followed through three successive backcross generations. J. Theor. Appl. Genet. 95: 895-902.

Haanstra, J. P. W. 2000. Characterization of resistance genes to Cladosporium fulvum on the short arm of the chromosome one of tomato. Ph.D. Diss., Wageningen University. Wageningen.

Huang, N., E. R. Angeles, J. Domongo, G. Magpantay, S. Singh, G. Zhang, N. Kumaravadivel, J. Bennett, and G. S. Khush, 1997. Pyramiding of bacterial blight resistance genes in rice: marker assisted selection using RFLP and PCR. J. Theor. Appl. Genet. 95: 313-320.

Lefort, B. M., F. Rodolphe, and A. Charcosset. 1990. New prospects for the genetic analysis of quantitative traits. The search for important loci. J. Biofutu R. 91: 30-37.

McNally, K. L. and M. A. Mutschler. 1997. Use of introgression lines and zonal mapping to identify RAPD markers linked to QTL.Mol-bred. J. Dordrecht. 3: 203-212. 
MOAC, 2005. Selected indicators of Nepalese agriculture and population. Government of Nepal. Ministry of Agriculture and Cooperatives, Agri-Business Promotion and Statistics Division. Gender Equity and Environment Devision. Kathmandu. Nepal.

Ojha, B. R. 2001. AFLP fingerprinting of tomato backcross populations. M.Sc. Thesis. Wageningen University and Research Centre. Wageningen. The Netherlands.

Ojha, B. R. 2003. Amplified fragment length polymorphism mapping of tomato backcross populations. J. Inst. Agric. Anim. Sci. $24: 21-28$.

Ojha, B. R. 2004. Reproductive and fruit characterisation of commercial genotypes of tomato(Lycopersicon esculentum Mill). NUTA Journal. 3(3) : $27-33$.

Paterson, A. H., E. S. Lander, J. D. Hewitt, S. Paterson, S. E. Lincoln, and S. D. Tanksley. 1988. Resolution of quantitative traits into Mendelian factors by using a complete linkage map of restriction fragment length polymorphisms. Nature 335, 721-726.

Shakya, S.M., A. Srivastav, S.M. Shrestha, R.B. Thapa and M..D. Sharma. 1993. Collection, characterisation, evaluation and maintenance of tomato germplasm during winter at Rampur, Chitwan. IAAS Research Reports (1992-1993 ). Institute of Agriculture and Animal Sciences, Rampur, Chitwan, Nepal.

Stam, P. and J. W. Van Ooijen. 1996. JoinMap Version 2.0: Software for the calculation of genetic linkage maps. Wageningen. The Netherlands.

Thoquet, P., J. Olivier, C. Sperisen, P. Rogowsky, H. Laterrot, and N. Grimsley. 1996. Quantitative trait loci determining resistance to bacterial wilt in tomato cultivar Hawaii7996. J. Molecular Plant Microbe Interactions. 9: 826-836.

Upreti, G. and R. Adhikari.1984. Heat tolerant tomato ( Lycopersicon esculentum Mill) genotype evaluation during summer in Chitwan, Nepal. J. Inst. Agric. Anim. Sci. 5: 59-70.

Upreti, G., R. Adhikari, and S.B. Gurung.1985. Performance evaluation of local tomatoes during winter in Chitwan, Nepal. J. Inst. Agric. Anim. Sci. 6: 113-124.

Van Beroo, R. 2000. Use of molecular markers in Plant Breeding. Ph.D. Diss., Wageningen University. Wageningen.

Vicente, M. C. D. and S. Tanksley, 1993. QTL analysis of transgressive segregation in an interspecific tomato cross. Genetics 134: 585-596.

Voorrips, R. E. 1999. Map chart. Plant Research International, The Netherlands.

Vos, P., R. Hogers, M. Bleeker, M. Reijans, T. Lee, M. Hornes, A. Frijters, J. Pot, J. Peleman, M. Kuiper, and M. Zabeau, M. 1995. AFLP: A new technique for DNA fingerprinting. J. Nucl. Acid. Res. 23: 4407-4414. 